\title{
The Effect of Loading on Reactive Market Power
}

\author{
Antonio C. Zambroni de Souza \\ EFEI - Grupo de Engenharia de Sistemas \\ CP 50 - 37500-000 Itajubá, MG, Brazil \\ zambroni@iee.efei.br
}

\author{
Fernando Alvarado \\ University of Wisconsin \\ Madison, Wisconsin, USA \\ alvarado@engr.wisc.edu
}

\author{
Mevludin Glavic \\ University of Tuzla \\ Tuzla, Bosnia \\ glavic@untz.ba
}

\begin{abstract}
The degree of market power (more precisely, the potential for market power obtained from a market concentration indicator such as HHI) increases as system stress increases. This paper illustrates the effect of system loading on the reactive power HHI for various locations. The paper also illustrates the use of sensitivity formulas to come up with measures intended to improve (reduce) the overall HHI of a system by judicious addition of reactive power resources. Better results in terms of reduction of market concentration as a result of reactive power are obtained when the voltage collapse characteristics of the system are considered. The concepts are illustrated using the 14-bus and 57 IEEE-test systems.
\end{abstract}

\section{Introduction}

Market power in almost all aspects of the electric power industry has become a point of major concern as a result of the recent efforts to deregulate the industry. One of the subtlest points concerning market power deals with reactive power. In order to preserve system security, adequate voltage regulation must be provided.

Reactive power provision was traditionally considered to be an integral part of the active power dispatch. Thus, it was never dealt with as a separable problem. Under a deregulated structure, however, reactive power generation must be managed in order to yield a good voltage profile and adequate system security. Most reactive power suppliers in a system are the same generators that are supposed to be competing in the deregulated energy market. Reactive power supplies can make some particular generator a "must run" unit (thus affording this generator effective market power). The problem becomes more complicated when contingencies are considered, since reactive power reserves are likely to be required for security reasons. A strategy for estimating the degree of reactive market power, which takes into consideration the changing operating characteristics of the system, is mandatory for an economically efficient competitive power system.
There are two alternative ways of estimating market power (or market concentration):

Simulation and analysis: In this case, one is interested in evaluating the impact on cost of withholding a particular commodity (in our case, the cost of withholding reactive power) $[1,2]$. An estimate of producer behavior on prices requires assumptions about tariffs, market development strategies, and the contractual framework within which the participants operate. This (more accurate) method for estimating market power is not used in this paper.

Reactive market concentration indices: In this case the focus is to determine how much the reactive market power is/is not concentrated. The idea is to determine the region of the system where additional sources of reactive power injection may be required. If such a region exists, reactive market power is presumed to exist. Otherwise, the reactive power is not concentrated, and reactive power market does not exist

This paper deals only with the second approach. The index used here to measure reactive market power is the $H H I[3,4,5]$. Section 2 illustrates how this index can be used to measure the degree of local reactive market power for each load bus in the system. As system conditions change, (in particular, as system stress and loading increases) the number of suppliers able to effectively deliver reactive power can change drastically. This paper also considers the association between market power and system load increase. In particular, it considers the effect on the $H H I$ of a system that operates in the vicinity of voltage collapse problems. This paper evaluates the $H H I$ for points along the voltage collapse path.

The paper is organized as follows: Section 2 reviews the definition of HHI. Section 3 shows how continuation methods can be used to trace a system under conditions of increasing stress, all the way to the "point of collapse." The results obtained in the paper for a sample system are shown in Section 4. Conclusions and implications are discussed in Section 5. 


\section{Computing the HHI}

Define the $H H I$ (Herfindahl-Hirschman Index) as:

$$
H H I=\sum_{i=1}^{N} s_{i}^{2}
$$

where $N$ is the number of market participants and $s_{i}$ is the percentage market share of each participant. An $H H I$ index value of 10,000 is an indication that the entire market is in the hands of a single supplier. $H H I$ values below 1,800 are generally considered an ab-initio indication of the absence of market power [5].

While the index itself may not always be the best indicator of market power, changes in the index (which are the main subject of this paper) convey useful information.

The relative ability of a generator to participate in the provision of voltage regulation at a bus (as measured by $s_{i}$ ) depends on both the influence of the generator reactive power on the desired voltage, and on the amount of reactive power available from the generator. The incremental ability of a generator to influence a voltage under specific operating conditions is defined by the Jacobian matrix.

Reactive power, like active power, must be balanced at all times. The Jacobian used in this paper is one in which the task of balancing active power is allocated to the slack generator (thus the row and column corresponding to this location are omitted). All other generators are presumed to be in PQ mode. This makes the generators adjust the voltage "one generator at the time," without the influence on voltages of other nearby generators. The exception is the slack generator: due to the need to balance not only active power but also reactive power, it is necessary to do something to attain reactive power balance. The most expedient thing to do is to let the slack bus also absorb any imbalances in reactive power. Unlike the active power case, this decision alters the behavior of voltages in the vicinity of the slack generator. Nevertheless, it yields better results than the alternatives, which are to either let all generators regulate $\mathrm{V}$ (thus masking the ability of a generator to adjust a remote voltage that is "behind" another generator) or results in reactive power imbalance (leading to matrix singularities and poor matrix behavior). ${ }^{1}$

Let the Jacobian constructed according to these rules

\footnotetext{
${ }^{1}$ The alternative of measuring "net MW" injections that net-out the controlling bus $\Delta \mathrm{Q}$ against the slack bus $\Delta \mathrm{Q}$ was considered but is not pursued here.
}

(rows are PQ for all buses except the slack, columns are values of $\theta$ and $\mathrm{V}$ for all buses except the slack) be $J$.

The reactive power capability of the individual generators also affects their ability to control voltage. Thus, the coefficient $s_{i}$ takes into consideration both the relative available reactive power from each of the generators in the system, and also take into account the impact that a generator can have on a specific voltage.

When defining the $H H I$ one can make two assumptions about the reactive power capability of the generator:

1. The capability of the generator is its total range of reactive power $Q^{\text {range }}=Q^{\max }-Q^{\text {min }}$.

2. The capability of the generator is the difference between the maximum reactive power capability and the current reactive power output $Q^{\text {margin }}=Q^{\text {max }}-Q^{\text {actual }}$.

The latter criterion measures the available margin. Market power develops under circumstances where only one generator (or a small subset) is able to influence a particular voltage because all other generators are either incapable of controlling the desired voltage, or have no remaining capability to do so. This renders the generator a "must run" unit. Under these conditions, the market power that develops affects not only the reactive power of the generator, but its active power as well. Generators that are classified as "must run" as a result of reactive power concerns are, for all practical purposes, monopoly generators.

If the $H H I$ is defined based on the entire range available to each generator, the changes to all voltages due to injections at generator $m$ is obtained from:

$$
\left[\begin{array}{c}
\Delta \theta \\
\Delta V
\end{array}\right]=\left(J^{-1} e_{i}^{Q}\right) Q_{i}^{\text {range }}
$$

where $Q_{i}^{\text {range }}$ is the reactive power range at generator $m$, and $e_{i}^{Q}$ is a vector of all zeros except for a nonzero in the position corresponding to the injection location $i$. (These values are not obtained from explicit inversion but rather from sparse forward and back substitution.) After evaluating $\Delta V_{i}$ for all participants $i$ in relation to the generators $m, s_{i}$ is computed from:

$$
s_{i}=100 * \frac{\Delta V_{i}}{\sum_{k=1}^{n_{g}} \Delta V_{i}}
$$


The $H H I$ is then computed from equation (1). As the number of generators increases, the value of the $H H I$ is reduced. From [5], a market free from market power concerns has an HHI of 2000 or less, whereas a monopoly is identified through an index approaching 10000 .

Instead of using the entire range of reactive power, it is possible to estimate reactive power market power indices using only the actual remaining reactive power margin for each generator. All that is needed is to replace $Q_{i}^{\text {range }}$ with $Q_{i}^{\text {margin }}$ in (2). The tendency of this action will be to result in a larger $H H I$ value. As a consequence reactive power may arise.

In the determination of reactive power market power it is important to consider all sources of reactive power, not just generators. This includes shunt capacitors and reactors at load buses. Shunt components play an important role on the system voltage profile, and are considered here.

\section{The Continuation method}

The continuation method is a numerical method that is used to trace the path of a power system from a stable equilibrium point up to a bifurcation point [6]. Such a method employs the following model:

$$
f(x, \lambda)=0
$$

where $\mathrm{x}$ represents the sate variables and $\lambda$ is a system parameter used to drive the system from one equilibrium point to another. Because a set of power flow equations suffices for such a model, it has been employed for voltage collapse studies, with been considered as the system load/generation increase factor.

Continuation methods are well covered elsewhere in the literature. Suffice it to say that the continuations methods rely on the choice of a continuation parameter (initially the degree of system loading $\lambda$ ) and on a twostep predictor-corrector iterative process:

The predictor step, used to indicate a direction to move. The tangent vector may be used for this purpose. It is given by:

$$
T V=J^{-1}\left[\begin{array}{l}
P_{0} \\
Q_{0}
\end{array}\right]
$$

where $J$ is the loadflow Jacobian and $P_{0}$ and $Q_{0}$ are the net active and reactive powers connected to each bus. The entries of the TV are in terms of angle changes $\Delta \theta$ and $\Delta \mathrm{V}$, scaled by $1 / \Delta \lambda$. Thus, the predictor step size to take is given by:

$$
1 /\|\mathrm{TV}\|
$$

where $\|$.$\| stands for tangent vector norm. Thus, the$ steeper the curve, the smaller the predictor step. It makes the method take bigger steps when the system is away from the bifurcation point, and smaller steps as the bifurcation is approached. The actual operating point is obtained with the help of the corrector stage, which is obtained from inclusion of an extra equation. This equation comes from the fact that the predictor and corrector vectors are orthogonal. As shown in [7], if the predictor step is given as an initial guess for a power flow program at the corrector point, the problem converges rapidly for a feasible operating point.

Using the continuation power flow, it is possible to solve the problem all the way to the maximum loadability point due to voltage collapse. More important, however, is that it is possible to determine the Jacobian matrix at this operating point. It is this matrix that will be most useful for purposes of selecting the locations where additions and changes to the system from the perspective of market power should take place.

\section{Test Results}

In this section, the IEEE-14 and 57 bus systems are used to test the ideas proposed in the foregoing sections. Two different $H H I$ definitions are compared: using the entire range to define the $H H I$, and defining the $H H I$ based on the available remaining capability.

From a base case, a continuation power flow is used. For each equilibrium point, the $H H I$ is computed for all load buses. The results obtained for bus 14 are depicted in Figure 1. No further operating point is possible, since the system reaches the voltage collapse point at a load factor of $2.0 \mathrm{pu}$.

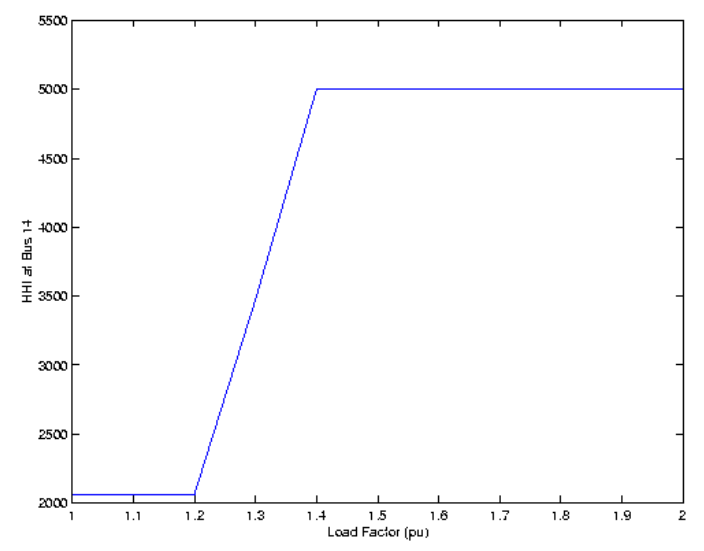

Figure 1: HHI at Bus 14 (entire regulation range of each generator and shunt devices) 
As the load increases, the HHI also increases. This result is expected, since voltage collapse problems arise as a consequence of lack of reactive power. Therefore, as the system approaches the voltage collapse point, the reactive market power offers an increasing demand.

Figure 2 presents results for the same system when the actual reactive power margin is considered. Once more, the index is evaluated for Bus 14. The index deteriorates more rapidly, as expected. Therefore, considering the reactive power effectively available in the system provides a more pessimistic (but perhaps more realistic) result.

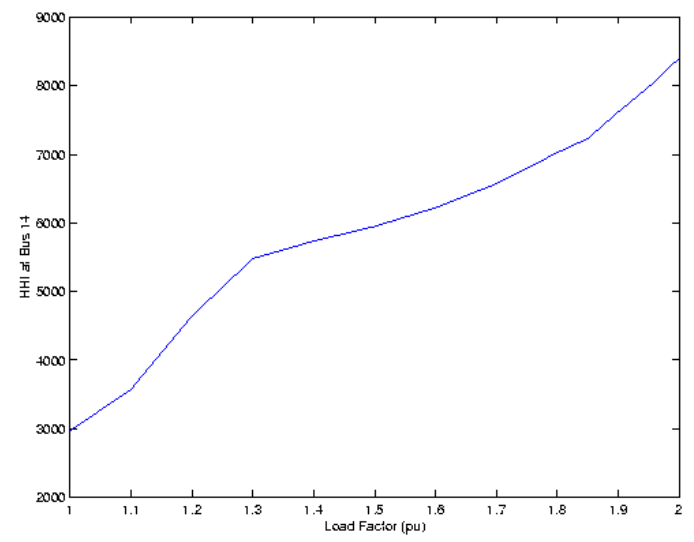

Figure 2: HHI at Bus 14 (using actual margin available of each generator and shunt devices)

A new test is now carried out, assuming that one wants to reduce the $H H I$ at as many locations as possible. The idea is to inject reactive power in the bus most effective from the point of view of reactive market power, i.e., that bus whose reactive power injection reduces the $\mathrm{HHI}$ for the largest number of buses. Figure 3 shows the $H H I$ values at the bifurcation point when the actual reactive power margin is considered.

In order to evaluate the suitability of locations to relieve market power conditions, two locations are compared: Bus 7, (associated with the worst $H H I$ ) and Bus 14 (the critical bus from the voltage collapse point of view). The tangent vector at the point of collapse was used to identify the critical bus. The interested reader is referred to [7] for details. In each case, the same amount of additional reactive power injection ( 0.5 $\mathrm{pu}$ ) was added to each bus. For each of these two cases, the problem was once again solved all the way to the point of maximum loadability (voltage collapse). At this point, the new values for the $H H I$ were computed. The results are shown in Figure 4. The dark bars are connected with case when shunt compensation at Bus 14 takes place. The gray bars provide the information for the case of reactive power addition at Bus 7.

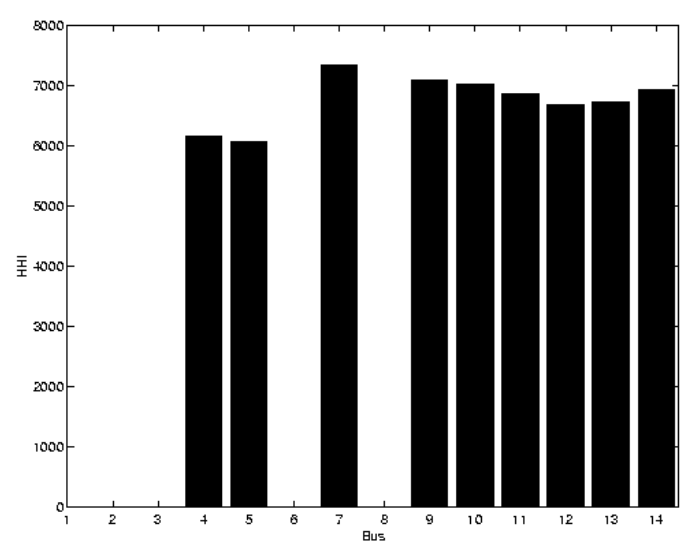

Figure 3: $H H I$ at the bifurcation point for every bus in the system (using actual margin)

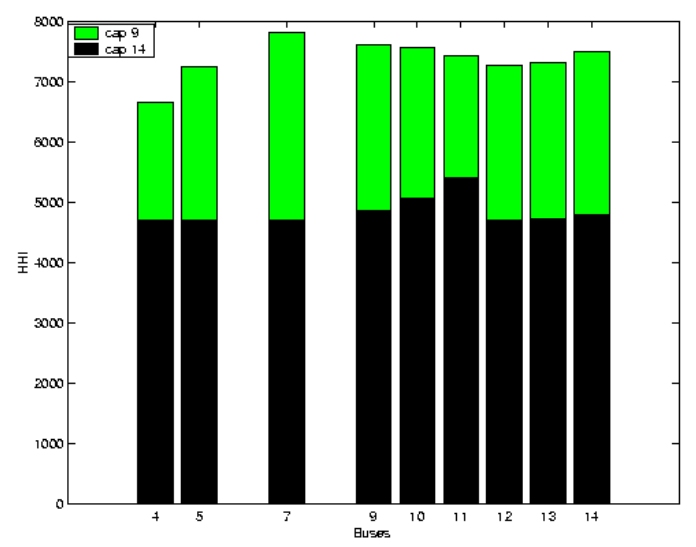

Figure 4: HHI with capacitor placement at the critical bus (solid) and at bus 7 (solid+gray).

Injecting reactive power at the critical bus provides an improvement in the $H H I$ for all the buses in the system. In contrast, attempting to control reactive market power by adding compensation to the bus that "seems" to have the worse reactive power problem is not nearly as effective. This important result drives one to conclude that voltage collapse analysis should be incorporated into any reactive market power analysis, and that the addition of reactive resources must likewise take into consideration market power issues.

The IEEE 57-bus system is used next. The tests take into consideration only the actual reactive margin at each generator. The results are depicted in Figure 5. Bus 31 is chosen for analysis, since it is the critical bus from the voltage collapse point of view.

The largest value of $H H I$ is seen at Bus 7. It is 7848 at the bifurcation point. A 0.2 pu capacitor is added (a $0.5 \mathrm{pu}$ capacitor is too large - it drives the system of equations to divergence), separately at each bus in turn. 
The dark bars are connected with case when shunt compensation at Bus 7 takes place. The gray bars provide the case of reactive power additions at Bus 31 . The buses illustrated along the horizontal axis are 7, 30, 31,32 and 47, respectively.

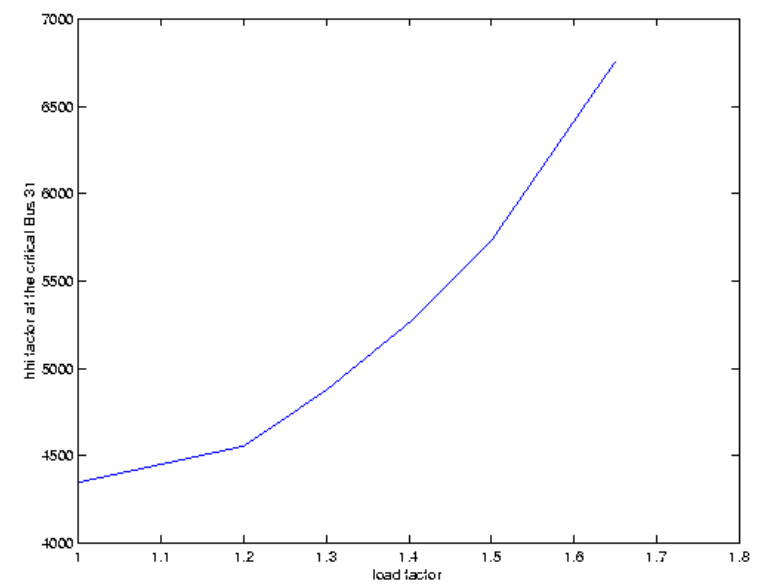

Figure 5: HHI at Bus 31 (using actual margin available of each generator and shunt devices)

As one can see from Figure 6, the same conclusions as those pointed out for the 14 bus-system are valid. The critical bus tends to be the valuable location in the system in a broader sense.

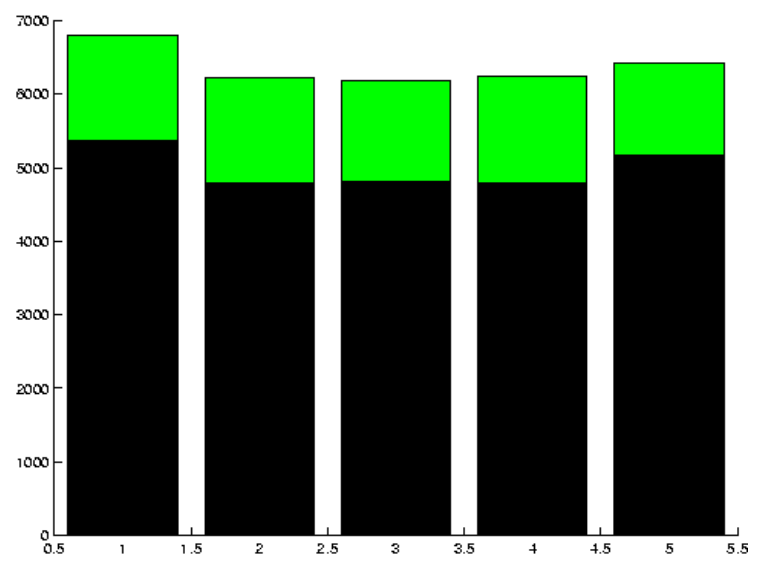

Figure 6: HHI with capacitor placement at the critical bus (solid) and at bus 7 (solid+gray).

\section{Conclusions}

The results obtained so far show that estimating reactive market power must take into consideration aspects related to voltage collapse. Reactive power injection at the system critical bus may improve the overall reactive market at the system, in contrast with reactive power injection at the bus associated with the worst reactive market index.
Therefore, determining the critical buses under the voltage collapse point of view may help one to provide an overall improvement in the reactive market conditions. This insight must be considered along with the fact that using the actual reactive reserve of each generator is crucial for reactive market estimation. In other words, in order to reduce reactive power market power it is better to address the fundamental voltage problems associated with voltage collapse conditions, rather than to attempt to in an ad-hoc way address specific instances of perceived reactive power induced market power.

Not described here is the possible interaction between Market Power in reactive power and the benefits it may bring about in terms of active power prices. However, it should be clear that a market power condition in reactive power can lead to a restriction of the active power market to a smaller set of contenders, as a result of the limits imposed on the transmission system from voltage concerns.

\section{Acknowledgements}

A. C. Zambroni de Souza thanks FINEP/RECOPE (project 0626/9 SAGE), CNPq, CAPES and FAPEMIG for financial support. Mevludin Glavic is the Fulbright Postdoctoral Scholar at University of WisconsinMadison, and he acknowledges support from Council of International Exchange of Scholars (CIES). Fernando L. Alvarado acknowledges partial support under NSF contract EEC-9815325 and the help of Ian Dobson.

\section{References}

1. S. Ahmed, G. Strbac, "A method for simulation and analysis of reactive power market," Proceedings PICA 1999, pp. 337-342.

2. A. Whitehead, M. Johnson, Solicitors, "A New Market in Reactive Power,"

http://www/ngc.co.uk/ancillary/mn_ancillary.html

3. T. J. Overbye, K. Patten, "Assessment of Strategic Market Power in Power Systems," PESS Winter Meeting January 1999, Vol. 1, pp. 297-302

4. F. Alvarado, T. Overbye, P. Sauer, "Measuring Reactive Market Power," Proceedings of the IEEE Winter Meeting 1999", January 1999, New York.

5. US Department of Justice FTC, "Horizontal Merger Guidelines," April 1992, Revised April 8, 1997.

6. R. Seydel, From Equilibrium to Chaos-Practical Bifurcation and Stability Analysis, Elsevier Science, North-Holland, 1988.

7. A. Zambroni de Souza, C. A. Cañizares and V. H. Quintana, "New Techniques to Speed up Voltage Collapse Computations Using Tangent Vectors," IEEE Transactions on Power Systems, vol. 12, No. 3, August 1997, pp. 1380-1387. 